\title{
Die Universitäten: »Im Kern verrottet《 oder das Beste was wir derzeit haben?
}

Der 23. April ist von der UNESCO zum »Tag des Buches« erklärt worden. Er wird weltweit mit Feiern begangen. Auch an der FU Berlin wurde 1996 des Tags des Buches, allerdings etwas schwermütig, gedacht. Denn just im April sind Bibliotheksetats der Universitäten um an die $50 \%$ zusammengestrichen worden, so daß viele Bibliotheken in diesem Jahr kein einziges Buch mehr werden erwerben können. Zeitungen und Zeitschriften werden abbestellt, was mittelfristig für viele wissenschaftliche Zeitschriften das »Aus« bedeutet, weil die Abos ausgehen. Die wissenschaftlichen Kommunikationsmöglichkeiten werden eingeschränkt. Die Vielfalt, für den wissenschaftlichen Evolutionsprozeß unverzichtbar, wird zur Einfalt, aus der die Sparpolitik ihre Begründung holt. Ebenso planlos und unüberlegt wie in der Phase des klotzigen Aufbaus einer olympischen Hauptstadt wird in Berlin nun zukunftsblind gespart. Am $\gg$ Rückbauk, wie der Abriß von Gebäuden heute schöngeredet wird, sind immerhin Architekten, Statiker und manchmal auch die Bürger beteiligt. Beim Rückbau der Universitäten entsteht der Eindruck, daß hier nur Bagger, Abrißbime und Betonköpfe in Funktion sind. Après nous le déluge.

$\mathrm{Da}$ in Berlin und anderswo gespart werden muß, weiß inzwischen jedes Kind. Kinder müssen sich nicht Rechenschaft über die Konsequenzen ihres Tuns ablegen, Politiker und Wissenschaftler aber sehr wohl, und nicht nur in Berlin. Dabei muß eine viel zu selten gestellte Frage aufgeworfen werden: Wie kommt es eigentlich, daß nach Jahrzehnten ungestümen wirtschaftlichen Wachstums in den Industrieländern, nach dem »Sieg im Kalten Krieg« über den »realsozialistischen « Systemwettbewerber, nach dem Triumph eines historischen Modells, zu dessen Bestandteilen auch die weitgehend öffentliche Finanzierung des Bildungssystem gehört, auf einmal die öffentlichen Kassen knirschend leer sind und weltweit die Sozial- und Bildungshaushalte unter dem Beifall selbstbewußter Konservativer und Liberaler zusammengestrichen werden? Es ist ja verwunderlich, daß Gesellschaften mit dem ökonomischen Wachstum immer reicher und trotzdem die Mittel für zukunftswichtige Bereiche immer knapper werden. Da stimmt etwas mit der Verteilung nicht, und da die Verteilung viel mit der Art und Weise der Produktion zu tun hat, kann wohl geschlußfolgert werden, daß die offenkundige Krise der Universität ohne Analyse der Gesellschaft nicht $\mathrm{zu}$ verstehen ist. Wenn die Universitäten als »im Kern verrottet《 gelten und dieses Urteil (von Dieter Simon) weithin zustimmend zur Kenntnis genommen wird, dann wundert es, warum es exklusiv auf eine wichtige gesellschaftliche Institution gemünzt wird und nicht diagnostisch danach gesucht wird, wo die Ursachen der Verrottung in der Gesellschaft insgesamt liegen. Diese Frage im übrigen war der Ausgangspunkt der inzwischen 
fast in Vergessenheit geratenen kritischen Hochschuldiskussion (SDSDenkschrift »Hochschule in der Demokratie «) und der »Kritik der politischen Ökonomie des Ausbildungssektors « der $60 \mathrm{er}$ und 70er Jahre, als sich die damalige Studentenbewegung daranmachte, die Krise der Ordinarienuniversität aufzuarbeiten und nach Ansätzen der Erneuerung der Universitäten ebenso wie der Gesellschaft zu suchen. Demokratische Reformen waren angesagt, die, darüber herrschte Einigkeit, nicht auf das Hochschulwesen zu beschränken waren.

In den 60er Jahren kam die Bildungsökonomie auf, in deren Verlauf feingesponnene »Manpower-Studien《 durchgeführt wurden. Das an den Universitäten »produzierte《 Angebot an Qualifikationen sollte der Nachfrage auf dem Arbeitsmarkt angepaßt werden. Also ging es vor allem darum, mit makroökonomischen, technokratischen Modellen die Entwicklung der für Wachstum im Systemwettbewerb notwendigen Qualifikationsstruktur zu erforschen und dann die Bildungsinstitutionen zu veranlassen, ihren 》Output《 entsprechend $\mathrm{zu}$ planen. Tatsächlich paßte diese Art der Bildungsplanung, wie sie von der OECD international $\mathrm{zu}$ koordinieren versucht wurde, in die Zeit: Planung war im Westen keineswegs »megaout《 wie seit der neoliberalen Wende der Thatcher und Reagan, sondern vollständig und breit akzeptiertes staatliches Steuerungsinstrument, das, wie Fritz Baade schrieb, »aus dem ursprünglichen geistigen Eigentum des Sozialismus übernommen« (Wettlauf zum Jahre 2000) worden ist - zum Besseren der kapitalistischen Gesellschaften. Vollbeschäftigung war erstrangiges wirtschaftspolitisches Ziel und das dominante theoretische Paradigma war der Keynesianismus. Doch die Versuche der makroökonomischen Steuerung des Bildungssystems sind trotz Vollbeschäftigung gründlich gescheitert, wenn man von der Gründung der Reformuniversitäten und der allgemeinen Expansion der Zahl der StudentInnen und von Personalstellen im Bildungswesen absieht.

Inzwischen hat sich das dominante wirtschaftspolitische Paradigma zur Mikroökonomie verschoben und die nationalstaatlichen Kompetenzen der Regulation sind in Zeiten der Globalisierung auf Maßnahmen der Strukturanpassung im globalen Wettbewerb reduziert. Kein Wunder, daß dieser $\mathrm{Pa}$ radigmenwechsel auch auf das Bildungswesen generell und die Universitäten speziell durchschlägt: Die Tendenzen der »Ökonomisierung《 der Universitäten, ihre unternehmerische Steuerung folgen der paradigmatischen Vorherrschaft der Mikroökonomie. Auch die Universitäten werden, wie alle anderen Bereiche auch, in der Tendenz aus dem Schutz nationalstaatlicher Regulation genommen und dem globalen Wettbewerb ausgesetzt. Die vorrangige Aufgabe ist unter diesen Bedingungen nicht mehr die Qualifikation für einen expandierenden Arbeitsmarkt oder ein Beitrag zur Demokratisierung der Gesellschaft, zu selbstbewußter Partizipation, zu emanzipatorischen Reformen, sondern ein möglichst meßbarer Beitrag zur Verbesserung der internationalen Wettbewerbsfähigkeit des jeweiligen Standorts. Wohlgemerkt, dies gilt in der Tendenz, ist aber in der BRD bislang keineswegs Realität. Wie weit sich diese Tendenzen bereits in einflußreichen Konzeptionen zur Hochschulentwicklung niedergeschlagen haben, läßt sich im Beitrag von Torsten Bultmann nachlesen.

In der Arbeitsmarktkrise haben mikroökonomische Steuerungssysteme jedoch unausweichlich irrationale Folgen. Denn Bildung ist ein »positionelles Gut«: Mehr und »höhere« Bildung ist kein Zertifikat für höher bewertete (und bezahlte) Jobs, wenn die hoch qualifizierten Arbeitsplätze nicht mehr 
sondern eher weniger werden. »Ab einer bestimmten Grenze«, so Fred Hirsch, der den Begriff des $\gg$ positionellen Gutes« populär machte, »verschlechtern sich die Nutzungsbedingungen eines Gutes, je verbreiteter dessen Gebrauch ist «. Viele besser qualifizierte Bewerber konkurrieren um wenige knappe Stellen. Die Folge ist ein wachsender Druck nach unten; höher Qualifizierte verdrängen die geringer Qualifizierten. Der Dr. Azubi ist inzwischen vorstellbar (Die Zeit, 1.8. 96). Die letzten »beißen die Hunde«, d.h. sie werden in die Chancenlosigkeit und d.h. in die strukturelle Arbeitslosigkeit weggebissen. Aus diesem Sachverhalt wird nun der Umkehrschluß gezogen, daß mehr Bildung eher vor Arbeitslosigkeit bewahre und bessere Jobchancen biete als eine geringere Bildung. Dieses Argument ist falsch, solange es nicht den positionellen Charakter des Gutes »Bildung« berücksichtigt. Wenn alle Theaterbesucher aufstehen, um besser über die Sitzenden hinwegsehen zu können, gewinnt keiner. Individuell jedoch kann sich niemand dem Druck entziehen, auch aufzustehen, wenn es der Nachbar bereits getan hat. Infolgedessen ist es für Studierende rational, die Universitätsausbildung auch dann zu absolvieren, wenn man gar keinen Beruf anstrebt, für den eine universitäre Bildung unbedingt notwendig wäre und jede Chance zu ergreifen, vor dem Studienabschluß einen erfolgreichen Job anzutreten.

Nun geht das mikroökonomische Konzept davon aus, daß Bildung individuell zurechenbares »Humankapital« darstellt. Dieses könne sich verwerten, also wie Geld- und Produktivkapital in Form von Profiten und Zinsen einen Einkommensstrom generieren. Marx mokierte sich über den auch zu seiner Zeit gebrauchten Begriff des »Humankapitals « mit der Bemerkung, das sei so, wie wenn man das Auge als das Kapital des Sehens begreifen wolle.
Doch so betrachtet ist Bildung einer Investition vergleichbar, der Investition in Finanzanlagen auf den Cayman Islands oder in eine neue Produktionsstät te in den neuen Bundesländern, für deren Zustandekommen jeder selbst und nicht der Staat verantwortlich ist. $\mathrm{Ob}$ sich die Investition freilich auszahlt, und darin liegt die Irrationalität dieses Arguments, hängt nicht mehr von individuellen Entscheidungen, sondern von den Interdependenzen zwischen Individuen $\mathrm{ab}$. Wenn alle in ihre Bildung und Ausbildung investieren, stellen sich alle auf die Zehenspitzen und - haben möglicherweise nichts davon, jedenfalls nicht das versprochene höhere Einkommen. Die Marktrationalität, die mit dem mikroökonomischen Paradigmenwechsel an den Universitäten dominant zu werden droht, erzeugt also im klassisch griechischen Sinne tragische Resultate: Die Unausweichlichkeit des individuellen Scheiterns, obwohl alle nach Erfolg streben und dabei das individuell Richtige tun, erzeugt jenes Unglücklichsein unserer Helden, von dem die griechische Tragödie handelt. Ein Heldenzyklus.

\section{II.}

Die Krise der Universitäten ist nur zu einem Teil mit dem »jobless growth« der vergangenen Jahrzehnte und den Konsequenzen für Ausbildung, Bildung und damit auch für die universitäre Lehre und Forschung zu erklären. Der "Sparschwachsinn« (Dieter E. Zimmer), dem die Universitäten neuerdings ausgesetzt sind, hat andere Ursachen. Eine davon ist die »Entkoppelung von monetärer und realer Akkumulation«, d.h. die in den beiden Jahrzehnten der finanziellen Deregulierung entstandene Dominanz der privaten Geldvermögen, für deren Bedienung immer mehr und in allen Ländern die öffentlichen Hände in die Pflicht genommen werden, so daß die öffentlichen Ausgaben unter Druck geraten. Paradoxerweise ent- 
spricht der ideologisch präferierten Deregulierung und Privatisierung von Vermögen eine umfassende Sozialisierung von Schulden. Das ungestüme Wachstum der privaten Geldvermögen ist von einem ebenso schnellen Anstieg der öffentlichen Schulden begleitet. In vielen zentralstaatlichen Budgets der OECD-Mitglieder und in den meisten Landesetats der Länder der BRD drückt inzwischen das »Sekundärbudget《 des Schuldendienstes auf das Primärbudget der Leistungen für Soziales, für Bildung und andere infrastrukturelle Einrichtungen. In der öffentlichen Debatte wird, wenn von Sparen die Rede ist, wie selbstverständlich das Sekundärbudget des Schuldendienstes ausgenommen. Es ist für Sparvorschläge, von denen es wimmelt, weil sich damit derzeit Politiker profilieren können, sakrosankt.

Diese Entwicklung hat zu einer sozialen Strukturveränderung der »postmodernen« kapitalistischen Gesellschaft beigetragen. Die reichen privaten Geldvermögensbesitzer haben vorrangiges Interesse an stabilem Geld in der internationalen Währungskonkurrenz. Dies ist nur zu haben, wenn die öffentlichen Budgets möglichst ausgeglichen sind, da nur so inflationistische Impulse und Abwertungstendenzen abgewehrt werden können. Der Spielraum für makroökonomische Beschäftigungs- und Sozialpolitik wird also eingegengt. $\mathrm{Zu}-$ gleich ist das Interesse der »verclubten« Gesellschaft der Geldvermögensbesitzer an öffentlich bereitgestellter Infrastruktur, an Sozialleistungen und öffentlichen Bildungsangeboten beschränkt. Denn sie können sich diese öffentlichen Leistungen in vielen Fällen besser und einfacher privat besorgen. Die Privatisierungstendenz der öffentlichen Bildungseinrichtungen wird so kräftig unterstützt. Was öffentlich nicht mehr finanziert werden kann, können Private übernehmen. So kommen würdelose Handlungen zustande, etwa
Bettelbriefe an private Geldgeber mit der Bitte, Zeitschriften, die gerade wegen des Sparschwachsinns abbestellt werden mußten, für Bibliotheken zu abonnieren, damit sie ihre Aufgabe wenigstens in Maßen erfüllen können. Wie in anderen gesellschaftlichen Bereichen auch, führt diese Tendenz dazu, daß öffentliche Räume verschwinden, daß Orte der demokratischen Auseinandersetzung privat eingezäunt werden, daß der Gesellschaft der Raum genommen wird, wo sie reflexiv zu sich selbst finden kann. Die Vermarktwirtschaftlichung ist also wie ein Mahlstrom, in dem die Gesellschaftlichkeit, die Demokratic untergehen. Darauf hat jüngst Benjamin Barber hingewiesen; die durch die Vermarktwirtschaftlichung bedingte Entdemokratiserung der höhren Bildung in den USA ist auch das Thema des Beitrags von Bruce Spear.

Die Privatisierung verlangt eine andere Logik, um nicht zu sagen: einen anderen Sinn der universitären Forschung und Lehre. Denn nun haben sich beide als Dienstleistungen auf einem Markt zu behaupten, der von privaten Nachfragern mit ihrer monetären Nachfrage bestimmt wird. Die Leistungen in Forschung und Lehre sind also Dienste wie Finanz-, Versicherungs- oder Tourismus- und Beratungsdienstleistungen auch. Sie sind Waren, deren freier Handel inzwischen durch das General Agreement in Trade and Services (GATS) weltweit reguliert wird. In diese Richtung weisen manche Tendenzen der universitären Entwicklung, wie Torsten Bultmann in seinem Beitrag zeigt. Sie haben mit den sozialstrukturellen Veränderungen des modernen Kapitalismus der Geldvermögensbesitzer zu tun. Wie in allen Fällen sozialstruktureller Veränderung geschieht dies nicht ohne heftigen Widerstand der Betroffenen, der Universitätsangehörigen aller Gruppen. Dabei geht es nicht nur darum, materiell abzuwägen, was zu gewinnen ist und wie hoch die Ver- 
luste sind. Man wird sich über den Sinn der Universität verständigen müssen, wenn Forschungsergebnisse und Bildung als Waren wie Würstchen oder Entertainment für einen Markt erzeugt werden, dessen einfache, »katallaktische« Regeln (F. A. von Hayek) das hoch-komplexe universitäre System auf einmal steuern sollen. Diese nehmen noch nicht einmal die (sub)systemspezifischen binären Codes von »wahr oder »nicht wahr« zur Kenntnis, sondern lediglich jene des Geldes: »to have or not to have «.

III.

Welchen gesellschaftlichen Nutzen können Universitäten an der Schwelle zum 21. Jahrhundert über die ihnen mehr und mehr zugewiesene Funktion als möglichst effizienter Dienstleistungsanbieter hinaus eigentlich haben? Auch auf diese Frage kann eine befriedigende Antwort nur gegeben werden, wenn Universitäten als Orte der Bildung, der Reflexion, der Expertise und (Politik)beratung in der Gesellschaft wahrgenommen werden. Die Zuweisung von Aufgaben dieser Art hat in der Geschichte eigentlich erst zu ihrer Gründung veranlaßt; darauf verweist mit Emphase Ekkehart Krippendorff und daran erinnert mit Blick auf Schleiermachers Programmschrift zur Gründung der Berliner HumboldtUniversität Christina Thürmer-Rohr. Heute sind Universitäten unverzichtbarer Bestandteil der »Expertensysteme« (Anthony Giddens), die jede moderne Gesellschaft braucht, um mit den sozialen, ökologischen, technischen, ökonomischen Risiken vorsorglich umgehen zu können. Dies mag der politischen Klasse und dem Wissenschaftsmanagement einleuchten, sofern die Naturwissenschaften oder die Medizin angesprochen sind.

Im Falle der Geistes- und Sozialwissenschaften hingegen herrscht erneut Ignoranz vor, nachdem der Schock der
》1000 Jahre« nach 1945, wie Christina Thürmer-Rohr für die Technische Universität Berlin zeigt, Geschichte ist, die in der Gegenwart kaum noch aufrührt. Doch »Expertensysteme《 sind funktional unvollständig, wenn die technischen und ökonomischen Risiken nicht als Elemente eines gesellschaftlichen Naturverhältnisses verstanden werden, als Ausdrucksformen von gesellschaftlichen Widersprüchen und Interessengegensätzen (so beispielsweise Egon Becker in PROKLA 79 oder die Ver. einigung deutscher Wissenschaftler in ihrer Kritik am Forschungsmemorandum der DFG von 1996). Eine bloße Dienstleistungsinstitution, die auf Marktsignale reagiert, ist zur Selbstreflexion, zur kritischen Beobachtung aller Handlungsfolgen, gerade wenn sie zeitlich und räumlich jenseits der Reichweite des Markthandelns liegen, im Prinzip nicht in der Lage. Die Dienstleistungsuniversität ist unter diesem Aspekt betrachtet eine armselige und gesellschaftlich riskante Institution, sie wirkt dabei mit, Gesellschaften kurzsichtig, ja blind zu machen, und die Fähigkeit zur Selbstbeobachtung verkümmern zu lassen. Die Risiken verschwinden freilich nicht, wenn die Aufgabenbe- und zuschreibung der Universitäten geändert wird; andere, neue Institutionen müßten diese sozial lebenswichtige Aufgabe übernehmen. Welche? Welche besseren gibt es als die guten, alten Universitäten, auch wenn diese sich derzeit in einer Krise befinden und tatsächlich reformiert werden müssen?

Die Wissensbestände und daher auch die Qualifikationen verändern sich mit atemberaubender Geschwindigkeit. Es ist noch nicht lange her, da war der Beruf des Schmieds oder des Müllers und Schlossers gleichzeitig Anlaß für den Familiennamen; man konnte vom Familiennamen auf die berufliche Identität zurückschließen. Das ist inzwischen ausgeschlossen. Daß heute 
ein allgemeiner Lehrstellenmangel herrscht, hat nicht nur mit der vorherrschenden mikroökonomischen Rationalität der Unternehmen zu tun, die sich um die makroökonomisch irrationalen Effekte ihres Tuns nicht kümmern, sondern auch damit, daß es den tradierten Beruf, den man vor Eintritt ins Berufsleben erlernt und dann ein Leben lang ausübt, um sich darin zu vervollkommnen und die Vervollkommnung an die nächste Generation weiterzugeben, kaum noch gibt. Ein Aspekt der Krise des Sozialstaats ist der Verlust der »Beruflichkeit«, wie Claus Koch und Karlheinz Geißler mehrfach dargelegt haben. Berufe haben Identität produziert; wie kommt diese zustande, wenn die Berufe sich in Jobs auflösen und lebenslanges Lernen von Wissensbeständen, die in spezialisierten Dienstleistungseinrichtungen unter dem Druck der Konkurtenz in immer schnelleren Zyklen ausgearbeitet werden, angesagt ist? Es wird die Dauerbereitschaft zur Weiter- und Umbildung erwartet. Die ganzen Volkswirtschaften verordnete »Strukturanpassung « mu $\beta$ auch individuell bis zur Charakterlosigkeit und bis zum Identitätsverlust nachvollzogen werden. Der fetischhaft subjektivierte Markt hat das Sagen, und alle müssen bedingungslos Folge leisten. Dieses Ansinnen in »unserer schnellebigen Zeit« erzeugt einen unerhörten Streß, dem vor allem diejenigen ausgesetzt sind, die über keinen soliden Wissensfundus und die Methodik der Wissensverarbeitung verfügen. Die generellen Qualifikationen, allgemeine Bildung oder »studium generale« erlangen Bedeutung nicht nur als unverzichtbares Element politischer Bildung, sondern für die Befähigung zum »lebenslangen Lernen«. Dieses ist keineswegs ein Wert an sich; im Gegenteil, es ist eher Ausdruck der ökologischen Krise unserer Zeit, in der Langsamkeit, natürliche Rhythmen mehr und mehr durch soziale Regeln der Beschleunigung überlagert und zugrundegerichtet werden. Statt Identitätsbildung ist daher Individualisierung der Weg, auf dem gegen die Tendenzen der Entropiesteigerung Resistenz ausgeübt werden kann. Daß diese Wandlung nicht bedeutungslos für die Hochschulen ist, dürfte offensichtlich sein.

IV.

Einem aufmunternden Artikel in der FAZ hat der Historiker Langewiesche eine Beobachtung vorangestellt, die wahrscheinlich alle Hochschulangehörigen bestätigen können: die Massenuniversität hat keineswegs in jeder Hinsicht einen Verfall der Lehre oder eine Verminderung der Qualität der Abschlüsse und der Forschungsleistungen zur Folge. Eine Vielzahl der Seminararbeiten, sei es im Grund- oder im Hauptstudium, auch die Diplomarbeiten und die Dissertationen, sind von einer Qualität, die von vergleichbaren Arbeiten in den 60er oder gar 50er Jahren, also in Zeiten der sguten alten Universität« selten erreicht worden ist. Offensichtlich hat die größere Masse derjenigen, die den Zugang zur Universität gefunden hat, auch dazu geführt, daß die Qualität von Abschlüssen gestiegen ist. Das ist nicht verwunderlich, wenn man evolutionstheoretische Überlegungen anstellt. Die Demokratisierung der Bildungschancen hat der Universität gutgetan. Diese positive Einschätzung des Bürgerrechts auf Bildung (Dahrendorf in den 60er Jahren) soll freilich nicht darüber hinwegtäuschen, daß Massenseminare und schlechte Betreuungsrelationen für so manche(n) Studentin/en auch Chancen mindert, die Lernfähigkeit begrenzt, der unpersönlichen Universität jenen prickelnden Lern- und Forschungseifer nimmt, der das ausmacht, was seit Platon als »pädagogischer Eros« bezeichnet wird. $\mathrm{Ob}$ diese gemeinsame die Studiensituation zu einer »studentischen Politik« führen kann, wird im 
Beitrag von Ralf Oberndörfer kritisch hinterfragt.

Nun wird auch eingewendet, die Massenuniversität habe zu einem beträchtlichen Anteil von drop-outs geführt. Das ist bei vordergründiger Betrachtung nicht falsch, aber auch nicht verwunderlich. Viele Studierende - es ist nicht bekannt, wie viele es tatsächlich sind -, die unter günstigeren Arbeitsmarktkonstellationen eine Lehre absolviert hätten oder einen anderen Weg der Berufsbildung gegangen wären, hätten vielleicht gar nicht die Universität besucht. Universitäten sind in der Krise der Arbeitsgesellschaft vergleichsweise billige Arbeitsmarktinstitutionen, in denen sich Jugendliche, die sonst ohne Arbeit und ohne Berufsaussichten auf der Straße oder in Arbeitsbeschaffungsmaßnahmen perspektivlos wären, bilden können. Weil die Arbeitsmarktchancen für Jugendliche in allen europäischen Ländern so schlecht sind, studiert im übrigen, ganz anders als in den glorreichen Zeiten der Vollbeschäftigung, ein immer größerer Teil eines Altersjahrgangs $(30-40 \%)$. Aus dem gleichen Grunde sind die Studienzeiten nicht kürzer und Studienabbrecher nicht unbedingt als erfolglos einzustufen, da sie häufig eine Gelegenheit mit Berufsperspektive ergreifen, die sie dazu veranlaßt, das Studium (lebensperspektivisch: eine intermediäre Phase der Arbeitsplatzsuche) zu beenden. Angesichts dieser realen Situation am Arbeitsmarkt wäre es sicherlich überlegenswert, die Fiktion aufzugeben, als ob es nur einen universitären Abschluß mit Magister, Staatsexamen oder Diplom geben könne, und nicht ordentliche Abschlüsse bereits nach der »Halbzeit« des Grundstudiums. Auch die Klage über die »unpolitischen« Studenten (zum politischen Bewußtsein vgl. den Aufsatz von Alex Demirovic), so begründet sie auch sein mag, greift zu kurz, wenn die Veränderungen des Arbeitsmarktes nicht berücksichtigt werden.
Die Demokratisierung des Bildungswesens hat zu einer paradoxen Situation geführt. Wegen der Engpässe des Arbeitsmarktes ergeben sich Krisentendenzen innerhalb des Institutionensystems der Universität, und darüber hinaus für ihr Selbstverständnis. Demokratisierung bedeutet, daß mehr Menschen als jemals zuvor über das »positionelle Gut« akademischer Bildung verfügen; daher verliert dieses für alle individuell an Wert, obwohl der soziale Nutzen einer breiten akademischen Bildung sehr hoch ist. Die breitere Massenbildung sollte also nicht negativ bewertet, sondern als eine Errungenschaft verstanden werden: Wenn alle in der Gesellschaft besser qualifiziert sind, kann dies für die »Ziviltät« der Gesellschaft nur positiv sein. Daraus folgt aber erstens eine Absage an elitäre Bestrebungen, die in der Debatte um die Universitätsreform als Suche nach der verlorenen $» E x-$ zellenz« viele konservative Hochschullehrer und Bildungspolitiker umtreibt. Dahinter steckt nichts als der Versuch., die Demokratisierungstendenzen des Bildungswesen, die von der SDSHochschuldenkschrift, von Picht, von Dahrendorf u.a. in den 60er Jahren begründet worden sind, rückgängig zu machen. Es ist allerdings noch nicht einmal effizienzsteigernd, das Rad zurückdrehen zu wollen. Peer Pasternak zeigt dies am Beispiel der Übernahme des westlichen Systems der universitären Verfassung in Ostdeutschland, wo die tatsächlich nach der Revolution von 1989/90 mögliche tiefgreifende Reform verpaßt worden ist, weil sich kurzfristige »vested interests" des hochschulpolitisch-politischen Komplexes westdeutscher im Prinzip konservativer Kreise haben durchsetzen können. Zweitens kann die zweifelsohne mit der Massenuniversität eingetretene Krise nicht gelöst werden, ohne Arbeitsmarkttendenzen und -politik zu verändern. Dabei geht es aber um Wirtschaftspolitik - und deren souveräne 
Gestaltung ist von den gleichen neoliberalen Politikern, die von der »verrotteten Universität« reden, an die globalisierten Finanzmärkte abgetreten worden.

V.

Die Einheit von Forschung und Lehre ist ein unverzichtbares Gut, und dies ist das unbestreitbar positive Element der Humboldtschen Idee von der Universität. Nur durch Forschung wird die Lehre interessant und wappnet sich gegen Langeweile. Von den Studierenden in der Lehre wiederum werden jene Fragen aufgeworfen, die des Forschers Naivität und Neugier bewahren. Beide sind unverzichtbar für tatsächlich innovative Forschungsergebnisse und eine gute und interessante Lehre. Die Einheit von Forschung und Lehre erzeugt jene Überraschungen, die Routine und Trott stören. Dies gilt im übrigen nicht nur für die Universitäten, deren konservative Eliten die Verbindung von Forschung und Lehre als Exclusivum für sich bewahren wollen, sondern gleichermaßen für die Fachhochschulen. Allerdings diskriminiert die derzeitige Praxis der Forschungsförderung die Lehre, ganz abgesehen davon, daß unter dem Stichwort der Elitebildung Lehre an der Massenuniversität und Forschung, die mit der Lehre möglichst wenig zu tun hat, im Zuge einer funktional-hierarchischen Arbeitsteilung differenziert zu werden drohen. Schon Forschungsanträge für Drittmittelprojekte sind dergestalt normiert (sowohl formaliter als auch hinsichtlich der Sprachregelung und des Horizonts, den sie abstecken), daß wirklich Neues selten zum Antrag gerät. Neues entwickelt sich daher - dies gilt zumindest für die Geistes- und Sozialwissenschaften eher außerhalb der formellen Drittmittelforschung. Dafür ist die Frauenforschung in ihrer Pionierphase ebenso Beleg wie ökologische Forschung, die sich aus den disziplinären und diszi- plinierenden Gleisen der Fachwissenschaften hinaus wagt. Die Forschungspraxis reproduziert die spath dependency« des wissenschaftlichen Fortschritts. Fortschritt auf anderen, neuen Pfaden ist nur schlecht oder gar nicht gefördert erreichbar. Das ist angesichts der globalen ökologischen Krise, der Migrationsbewegungen, der Transformationsprobleme, der Arbeitsmarktkrise etc. eine höchst problematische $\mathrm{Be}$ schränkung der Wissenschaften. Tatsächlich muß die Universität ihre soziale Aufgabe immer auch damit legitimieren, daß sie auf diese und viele andere Fragen Antworten auf der Höhe der Zeit zu geben vermag, insbesondere dann, wenn Wissen schnell veraltet.

Auf den eingefahrenen Pfaden haben sich häufig genug auch Machtkomplexe gebildet, die das wissenschaftliche System in fatale Richtungen steuern: der medizinisch-technische Komplex oder juristisch-ökonomische Beratungskartelle, energie- und materialwirtschaftiiche Komplexe, die ethisch-politischen und -wirtschaftlichen Rechtfertigungsmann- und frauschaften etc. Diese steuern sich selbst, sie bemessen sich wechselseitig an dem für die Bestandteile des Komplexes jeweils nützlichsten Resultate. Ein instruktives, nicht immer anregendes Beispiel sind die sthink tanks « in den USA, wie $F_{\text {rank }}$ Fischer in seinem Beitrag zeigt. In der Wissenschaft zählen Drittmittel-Einwerbungen als positives Kriterium, in der Wirtschaft jene wissenschaftlichen Resultate, die möglichst problemlos in Wert gesetzt werden können. Dazu passen kritische Wissenschaften nicht oder nur dann, wenn ein so dimensionierter Komplex in eine offenkundige Krise der Evolution geraten ist, d.h. wenn die Wissenschaft nicht mehr weiter weiß und das System des Profitmachens ins Stocken gerät, wenn sich die Komplexe also nicht mehr wechselseitig helfen können. Dann sind sogar kritische Innovatoren gefragt, so wie im Umbruch 
der 60er und 70er Jahre, als die kritische Intelligenz durchaus »transformistisch« (Gramsci) integriert worden ist, um das System insgesamt auf neue Pfade der Entwicklung zu schleusen. Dort, wo dies, wie in den Ostblockländern, nicht geschehen ist, war denn auch Stagnation die Folge.

Die heute verlangten Innovationen sollen aber nicht an einer Bifurkation der Entwicklung auf einen neuen Pfad verhelfen, sondern die internationale Wettbewerbsfähigkeit des Standorts im globalen »Wettlauf der Besessenen《 (Krugman) heben. Die Universitäten sollen sich am Output der verwertbaren Forschungsergebnisse und der produzierten Qualifikationen bemessen lassen. Damit sind wir erneut beim Stichwort Ökonomisierungsdruck, unter dem die Universität seit Jahren leidet und der derzeit durch den auferlegten Sparzwang zum Unerträglichen gesteigert worden ist. Die Versuche, die Universität betriebswirtschaftlich unter Effizienzkriterien zu managen, implizieren Selektionsentscheidungen, deren Kriterien nicht immer wissenschaftlich nachvollziehbar sind: Der eine Bereich wird gefördert, der andere nicht; der eine Studiengang gestrichen, der andere ausgebaut. Daß diese Selektion den politischen Schablonen folgt (etwa Diskriminierung der Frauenforschung), ließe sich an vielen Beispielen zeigen.

Die Selektion ist vom Prinzip her fragwürdig, weil der Output der Universität quantitativ im Prinzip nicht meßbar ist. Die Versuche, dies dennoch zu tun, sind so lächerlich wie die Vermessung der britischen Küste - darauf hat Benoît Mandelbrot verwiesen - ohne Verständigung auf Maßstab und Methoden und darüber kann man sich lange streiten. Eine Antwort kann es erst dann geben, wenn wir uns (a) über das Messgerät verständigen, ob wir (b) in Meter, Kilometer oder Zentimeter etc. messen, (c) entscheiden, ob bei Ebbe oder Flut gemessen werden soll, (d) definieren, welche Details wir nicht bemessen, welche aber sehr wohl berücksichtigen wollen. Und tatsächlich »hat die Küste Großbritanniens die gleiche Länge wie die von Manhattan oder von ganz Amerika. Sie sind alle unendlich lang« (Briggs/Peat 135f). Die derzeitigen Kriterien der universitären Küstenvermessung - Finanzvolumen von Drittmittelprojekten, Zahl der Publikationen, der Dissertationen, der Diplomarbeiten, der Konferenzen etc. - verselbständigen sich $\mathrm{zu}$ einem absoluten Maßstab, mit dem nicht indizierte und möglicherweise gar nicht indizierbare Leistungen gemessen werden sollen. Die für synergetische Effekte unverzichtbare Liebenswürdigkeit eines Kollegen, die gruppendynamisch positive Wirkung einer Kollegin können nicht oder nur falsch gemessen werden. Die »indizielle« Individualisierung der Leistung löst die Universität als sozialen Forschungs- und Lehrzusammenhang in eine Landkarte von eifersüchtig bewachten Schrebergärten auf, die sich bestenfalls mit Gartenzwergen schmükken. Die Versuche, Kriterien und Indizes wissenschaftlicher Leistungen festzulegen, zeigen sehr schön die Absurdität des Unterfangens, den Pudding wissenschaftlicher Leistungen an die Wand zu nageln. Nun kann man sich auf die Maßstäbe des Messens festlegen und so durchaus vergleichbare Resultate erreichen, die der Rationalisierung des »verrotteten« Universitätsbetriebs dienen mögen. Aber ob die Küste des Leistungseilands damit angemessen bemessen worden ist, bleibt dahingestellt. Schließlich wird man entdecken, daß der Schüler mit der mangelhaften Mathematiknote im Abitur zu einem der größten Physiker des Jahrhunderts mutiert, und daß aus vermeintlich totem Holz ein grüner Reis erwächst. Dieses Problem im Großen schürzt sich bei der Benotung jeder Klausur, Seminar- oder Magisterarbeit im Kleinen: eine gerechte Benotung ist nicht möglich. Man 
kann sich allenfalls über einen Rahmen verständigen.

Der äußere Druck führt so manches Mal zu einem internen Kannibalismus. Mobbing ist auch im Universitätsbetrieb kein Fremdwort mehr. Solidarität und Reziprozität sind nicht die Prinzipien der Kommunikation an der Universität; die von außen drängende Ökonomisierung hat ineffiziente Kämpfe um knappe Ressourcen ausgelöst. »Leistung « zu erbringen ist ja eine Selbstverständlichkeit; diese aber mit absurden Maßstäben bewertet zu wissen und die Abhängigkeit der Mittelzuweisungen von den Resultaten fragwürdigen Messens hat den aus der »Standortdebatte bekannten $»$ Wettlauf von Besessenen« zur Folge: Die Uni-Angehörigen wechseln vom Elfenbeinturm ins Mäuserad. Die Grenze dessen, was zivilgesellschaftlich gerade noch tragfähig ist, wird erreicht.

An diesem grundsätzlichen Dilemma der Quantifizierung wissenschaftlicher Leistungen bricht sich auch die in jüngster Zeit von den Wissenschaftsexperten vorgebrachte Forderung nach mehr Wettbewerb im Wissenschaftssystem. Damit es überhaupt Wettbewerb zuläßt, muß das Wissenschaftssystem als Markt organisiert werden, auf dem verschiedene Anbieter zu unterschiedlichen Konditionen vergleichbare Leistungen offerieren. Ihre Angebote sind dann wie diejenigen von Finanz-, Transport- oder Versicherungsdienstleistern zu gestalten. Doch die Frage ist, ob das geht. Denn Anbieter und Nachfrager universitärer Dienste dürften es in der Regel sehr schwer haben, die eigentliche Dienstleistung von den außermarktmäßigen Effekten (synergetische Effekte; urbanes Ambiente; Tradition) abzugrenzen. Ökonomisch betrachtet verkauft eine Universität ihre Dienstleistung immer als Kuppelprodukt zusammen mit anderen Leistungen, für die sie nur zu einem Teil verantwortlich zeichnet. Umgekehrt er- bringt sie Dienste, die überhaupt nicht zu Markte getragen werden können, weil diese Dienste öffentliche Güter par excellence sind: die Kultur und der Ruf einer »Universitätsstadt « zum Beispiel. Bei der Output-Messung ist diesem Sachverhalt kaum Rechnung zu tragen. Darin kommt fast als eine nostalgische Erinnerung doch zum Ausdruck, daß Wissenschaft, Forschung und Lehre, Bildung öffentliche Güter und nicht privat und individuell verwertbare $\mathrm{Wa}$ ren sind. Die Privatisierung und Transformation der Universitäten in Dienstleistungsanbieter ist mithin Ausdruck der von Bruce Spear gekennzeichneten Tendenz der »Entdemokratisierung «. Wenn Dienstleistungen verkauft werden, haben die Dienstleistungskäufer natürlich das Recht, Bestleistungen, Qualität zu verlangen. Die Konsumenten sind souverän. Wenn Studenten Studiengebühren entrichten, haben sie ein Anrecht darauf, entsprechend des fachspezifischen Standards zum gesellschaftlich zertifizierten Abschluß gebracht zu werden. Die Dienstleistungsuniversität muß diesem Anspruch wie andere Dienstleister anderer Branchen auch genügen, wenn der Dienstvertrag zustandegekommen ist. Sie muß daher erstens in der Lage sein, nicht jeden Nachfrager zu akzeptieren, da der wahrscheinliche Aufwand zur Leistung des Dienstes kalkuliert werden muß. Die marktwirtschaftliche Rationalität verlangt die Gegenüberstellung von voraussichtlichem Aufwand und realistisch erwartbarem Ertrag. Dies heißt also mit Notwendigkeit erstens die Einführung universitärer Eingangsprüfungen; die schulische Abgangsprüfung, das Abitur, ist dazu ungeeignet. Zweitens muß der Kunde (die Studentin/ der Student) eine Garantie haben, daß die bezahlte Dienstleistung zum versprochenen Erfolg führt. Die Kundenorientierung hat zur Folge, daß viele Leistungen, die Universitäten selbstverständlich erbringen, entfallen müssen: 
jene Bildungsangebote, die extrafunktional und im Sinne der Investition in Humankapital schlicht überflüssig sind. Es sei denn, dafür entsteht eine Nachfrage so wie im kommerzialisierten Rundfunk die Nachfrage nach einem kommerziellen Klassiksender entsteht. Die Vermarktwirtschaftlichung der Universität wird diese Institution ebenso vereinseitigen und ihrer Vielfalt berauben wie andere Bereiche auch, die der Macht des Marktes ausgesetzt sind. Ein Studium generale, für das sich $E k$ kehart Krippendorff stark macht, hat in einer Dienstleistungsuniversität keinen Platz, es sei denn als kommerzialisierte Wissenschafts-Show, in der der Professor als Entertainer fungiert.

VI.

Daß lebendige und daher auch rebellische Universitäten für das kulturelle Leben der jeweiligen Stadt ein Jungbrunnen sind, haben die vergangenen 30 Jahre gezeigt. Das Berlin des Jahres 1996 ist nicht vorstellbar ohne die Auswirkungen der Studentenbewegung der $60 \mathrm{er}$ Jahre. Das werden selbst jene zugeben, die 1968 Rudi Dutschke jagten. Dieses Innovationspotential, auch wenn die Formen, in denen es sich artikuliert, dem Bürger manchmal nicht passen, im kleinkarierten Sparen kaputtzumachen, ist nur Leuten möglich, die die Welt mit Lesebrillen wahrnehmen, die sie freilich hauptsächlich benutzen, um Parteibücher zu studieren. Aber die Aufgaben der Universität gehen darüber hinaus. Eine Gesellschaft braucht Institutionen, die der Reflexion des Entwicklungsweges fähig sind. Sonst werden die Regeln des $\gg$ Wettlaufs der Besessenen« die Gesellschaften einen Pfad entlangtreiben, auf dem der crash zwar vorhersehbar und daher auch vermeidbar ist, aber wegen der selbstverordneten Blindheit mikroökonomisch bornierter Interessen nicht vorhergesehen wird, geschweige denn, daß Vorsorge getroffen würde. Gerade in der tiefen sozialen und ökologischen Krise am Ende des Jahrhunderts haben die Universitäten tatsächlich eine Aufgabe: Alternativen zu erarbeiten, sich einzumischen, Kriterien und Visionen einer Zukunft aufzustellen, die den Bedingungen der Nachhaltigkeit - ökologisch und sozial - genügen.

In der Krise der Universität der 60er Jahre wurde, gerade an der FU, die Kritische Universität erfunden - mit der Absicht, die Wissenschaft zu emeuern, Bedingungen der gesellschaftlichen und individuellen Emanzipation theoretisch zu begründen und praktisch umzusetzen. Das ist ein auch heute wieder aktuelles Programm. Es geht um eine zukunftsorientierte Universität, den Prinzipien der sozialen Solidarität und der ökologischen Nachhaltigkeit verpflichtet - und der Kritik einer Gesellschaft und einer Wissenschaft, die sich der vom Markt verlangten Kurzsichtigkeit des schnellen Gewinns, des ungebremsten Wachstums, der rasch verwertbaren wissenschaftlichen Resultate, des schnellen Durchschleusens von Studentengenerationen für einen nicht vorhandenen Arbeitsmarkt verschrieben haben. Dafür lohnt ein Einsatz, dazu auch dieses PROKLA-Heft.

$* * *$

Eine Anmerkung in eigener Sache: Am 8. Juni 1996 fand in Berlin die Mitgliederversammlung der "Vereinigung zur Kritik der politischen Ökonomie e.V.« statt, die die PROKLA herausgibt. Dabei wurde auch die Redaktion gewählt. Aus der bisherigen Redaktion stand unser langjähriges Mitglied Kurt Hübner für eine erneute Kandidatur leider nicht mehr zur Verfügung. Neu in die Redaktion gewählt wurde Dorothea Schmidt. Erstmals sind nun mehr Frauen als Männer in der Redaktion der PROKL A vertreten. 